Discussion Paper No. 02-48

\title{
Why Are Asset Returns Predictable?
}

\author{
Erik Lüders
}

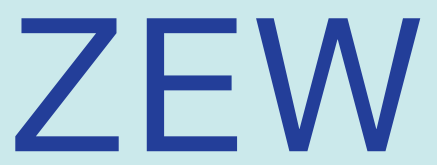

Zentrum für Europäische Wirtschaftsforschung GmbH

Centre for European Economic Research 
Discussion Paper No. 02-48

\title{
Why Are Asset Returns Predictable?
}

\author{
Erik Lüders
}

Download this ZEW Discussion Paper from our ftp server:

\section{ftp://ftp.zew.de/pub/zew-docs/dp/dp0248.pdf}

Die Discussion Papers dienen einer möglichst schnellen Verbreitung von neueren Forschungsarbeiten des ZEW. Die Beiträge liegen in alleiniger Verantwortung der Autoren und stellen nicht notwendigerweise die Meinung des ZEW dar.

Discussion Papers are intended to make results of ZEW research promptly available to other economists in order to encourage discussion and suggestions for revisions. The authors are solely responsible for the contents which do not necessarily represent the opinion of the ZEW. 


\section{Non-Technical Summary}

This paper analyzes how investors' risk-preferences influence asset prices. It is shown that nonconstant relative risk aversion leads to predictability in asset returns, although the market is informationally efficient. It is also shown that in this case asset returns are serially correlated. Thus, although the market is efficient and all investors act rationally, it is still possible to predict future returns to a certain extent.

Moreover, the analysis shows that the widely used financial ratios as the price-earnings ratio and the book-to-market ratio have predictive power for future returns. Hence, the paper provides an explanation for possible predictability of asset returns in a rational and efficient market. 


\title{
Why Are Asset Returns Predictable? ${ }^{1}$
}

\author{
Erik Lüders ${ }^{2}$
}

First draft: January 2002

This version: July 2002

${ }^{1}$ I am indebted to Günter Franke for invaluable support. I am also grateful for helpful discussions with Axel Adam-Müller, Jens Jackwerth, Bernhard Peisl, Michael Schröder, Susanne Warning and seminar participants at the Centre for European Economic Research, Mannheim, and the Center of Finance and Econometrics, Konstanz. All remaining errors are my sole responsibility. Financial support by the CoFE, Konstanz, and the ZEW, Mannheim, is gratefully acknowledged.

${ }^{2}$ Center of Finance and Econometrics (CoFE), University of Konstanz and Centre for European Economic Research (ZEW), Mannheim. Address: University of Konstanz, Fach D 147, 78457 Konstanz, Germany. Phone: ++49-7531-882541. email: erik.lueders@uni-konstanz.de 


\begin{abstract}
Starting from an information process governed by a geometric Brownian motion we show that asset returns are predictable if the elasticity of the pricing kernel is not constant. Declining [Increasing] elasticity of the pricing kernel leads to mean reversion and negatively autocorrelated asset returns [mean aversion and positively autocorrelated asset returns]. Under nonconstant elasticity of the pricing kernel financial ratios as the price-earnings ratio have predictive power for future asset returns. In addition, it is shown that asset prices will be governed by a time-homogeneous stochastic differential equation only under the constant elasticity pricing kernel. Hence, usually asset price processes do not satisfy the assumptions needed for empirical estimation.
\end{abstract}

JEL classification: G12

Keywords: Pricing kernel; Diffusion processes; Stationarity; Predictability of asset returns; Autocorrelation 


\section{Introduction}

In spite of the vast literature on deviations from the random walk hypothesis, there is still controversy whether these deviations are attributable to inefficient markets. ${ }^{1}$ Even those who favor the efficient market hypothesis do not agree whether these financial market phenomena point out to a new model of investor behavior or whether modelers should remain within the traditional framework of rational expectations and von Neumann-Morgenstern utility functions. There is no doubt that the behavioral finance literature has contributed much to our understanding of asset prices. ${ }^{2}$ However, it is highly controversial to what extent new behavioral postulates should be adopted. ${ }^{3}$

Surprisingly, although many models have been proposed for the explanation of asset returns, still little is known on how return characteristics change when the elasticity of the pricing kernel is not constant. Since throughout the paper we assume monotonic elasticity of the pricing kernel, i.e. either monotonic increasing or declining elasticity, our approach is consistent with the traditional framework of rational expectations and von NeumannMorgenstern utility functions.

On the basis of a parsimonious model for asset price processes this paper analyzes whether predictability can be explained by nonconstant elasticity of the pricing kernel. The results show that predictability may indeed be induced by nonconstant elasticity of the pricing kernel. More precisely, we show that asset returns are autocorrelated and financial ratios have predictive power for future asset returns. Since this paper shows what kind of return characteristics can be explained in the traditional framework, the paper provides a benchmark for the necessity of new behavioral postulates.

The economy considered in this paper is very simple. Following the approach of Franke, Stapleton and Subrahmanyam [18] we start from an exogenously given information process which characterizes investors' expectations about the asset price at terminal date $T$. The information process which describes investors' expectations is assumed to be governed by a geometric

\footnotetext{
${ }^{1}$ For an overview on deviations from the random walk hypothesis see for example Cochrane [12].

${ }^{2}$ For recent advances in behavioral finance see for example Barberis and Huang [2], Barberis, Huang and Santos [3], Barberis, Shleifer and Vishny [4] or Daniel, Hirshleifer and Subrahmanyam [14].

${ }^{3}$ See for example Brennan [9] for a discussion of the pros and cons of adopting new behavioral postulates.
} 
Brownian motion with constant instantaneous volatility and no drift. Hence, the information process is consistent with rational expectations. Furthermore, we assume that the pricing kernel is a deterministic function of the asset price which is perfectly consistent with a representative investor economy (e.g., Decamps and Lazrak [15]). Hence, our model is consistent with the model of Black and Scholes [8] except that we do not assume constant elasticity of the pricing kernel. The simplicity of the framework allows to show more clearly the effect of alternative assumptions on the pricing kernel.

Our analysis is related to the literature on the viability of asset price processes in a representative investor economy. ${ }^{4}$ Especially, this paper is closely related to Franke, Stapleton and Subrahmanyam [18] and to Lüders [24]. In both papers a similar economy is considered. However, Franke, Stapleton and Subrahmanyam [18] focus on option prices. While Lüders [24] proposes an alternative explicit price process to the geometric Brownian motion, the analysis in this paper is more general, since Lüders [24] assumes HARA utility. Our paper is also related to Johnson [23] who proposes a rational explanation for momentum on the firm level. While we focus on the elasticity of the pricing kernel as explanation for positive and negative serial correlation of asset returns, Johnson [23] unveils stochastic expected growth rate as an explanation for momentum.

The organization of the paper is as follows. Section 2 presents the model. In Section 3 the stochastic differential equation governing the equilibrium asset price process is analyzed for declining and increasing elasticity of the pricing kernel. Section 4 analyzes the implications for discretely sampled asset returns. Section 5 concludes.

\section{The model}

In this paper we consider a market with a given time horizon $T>0$ and the one-dimensional standard Brownian motion $W$ on a given filtered probability space $\left(\Omega, \mathcal{F}, \mathcal{F}_{t}, P\right)$ where $\left(\mathcal{F}_{t}\right)_{t \in[0, T]}$ is the filtration generated by $W$ augmented by all the $\mathcal{F}$-null sets, with $\mathcal{F}=\mathcal{F}_{T}$. It is assumed that at least one risky asset is traded and hence the market is complete. Moreover, we assume that the company does not pay any dividends until terminal date $T$. The distribution of the terminal value of the asset is exogenously given.

\footnotetext{
$[27]$.

${ }^{4}$ See Bick [5] and [6], Franke [17], He and Leland [20] and Stapleton and Subrahmanyam
} 
Following Franke, Stapleton an Subrahmanyam [18] and Lüders and Peisl [25] we define the distribution of the terminal value implicitly by postulating some information process. Such an information process can be interpreted as the representative investor's conditional expectation about the terminal value of the stock. The information process $I$ is defined by

$$
I_{t}=E\left(X_{T} \mid \mathcal{F}_{t}\right), \quad 0 \leqslant t \leqslant T,
$$

where $X_{T}$ is the random payoff of the stock at the terminal date $T$. Moreover, to keep things simple and to focus on the pricing kernel, we assume that this information process $I$ is characterized by a geometric Brownian motion with constant instantaneous volatility $\sigma$ and no drift, i.e.

$$
\begin{aligned}
d I_{t} & =\sigma I_{t} d W_{t}, \quad 0 \leqslant t \leqslant T \\
I_{0} & >0 .
\end{aligned}
$$

Note that with this assumption $I_{T}$ is lognormally distributed with

$$
\operatorname{Var}\left(\ln I_{T} \mid \mathcal{F}_{t}\right)=\sigma^{2}(T-t), \quad 0 \leqslant t \leqslant T,
$$

and

$$
E\left(I_{T} \mid \mathcal{F}_{t}\right)=I_{t}, \quad 0 \leqslant t \leqslant T .
$$

Thus, the information process is a martingale which is a necessary condition for rational expectations of the representative investor. Note also that with rational expectations, at terminal date $T$ the expected value of the asset $I_{T}$ and the price of the asset $F_{T}$ must be equal, i.e. $I_{T}=F_{T}=X_{T}{ }^{5}{ }^{5}$ To focus on risk preferences we always consider forward prices instead of spot prices.

Finally, we want to introduce a formal definition of what we mean by predictability of asset returns: ${ }^{6}$

Definition 1 Asset returns $r_{t, \tau}=\ln \frac{F_{\tau}}{F_{t}}$ are predictable if $\frac{\partial}{\partial x} R\left(t, \tau, x_{t}\right) \neq 0$, where the function $R\left(t, \tau, x_{t}\right)$ characterizes the expected return, i.e. $R\left(t, \tau, x_{t}\right) \equiv$ $E\left(r_{t, \tau} \mid \mathcal{F}_{t}\right)$ for all $0 \leqslant t \leqslant \tau \leqslant T$, and $x_{t}$ is some state variable.

With this definition asset returns are predictable if the expected return depends on some (observable) state variable. While predictability of asset

\footnotetext{
${ }^{5}$ For a detailed discussion of information processes see Lüders and Peisl [25].

${ }^{6}$ For a function $H: x \rightarrow H(x), \frac{\partial}{\partial x} H\left(F_{t}\right)$ has the same meaning as the widely used, but informal, notation $\frac{\partial}{\partial F_{t}} H\left(F_{t}\right)$. The more formal notation will be used in this paper.
} 
returns may manifest itself in different forms, we believe that this definition is a natural way of thinking about predictability, especially in our Markovian continuous-time setting. In Section 3 the forward price $F_{t}$ itself and the expected value of the terminal forward price $I_{t}$ will be used as state variables. In Subsection 4.2 we introduce an additional stochastic process which characterizes the book value of equity. The exogenous book value of equity will also be used as a state variable.

After the description of the information structure in the economy let us turn to the characterization of the pricing kernel. It is well known that in an arbitrage-free market an equivalent martingale measure exists. Moreover, in complete markets the equivalent martingale measure $\widetilde{P}$ is unique. The transformation from $P$ to $\widetilde{P}$ is given by the pricing kernel $\Phi_{t, T}=\frac{\Phi_{0, T}}{\Phi_{0, t}}$ where $\Phi_{0, t}=E\left(\Phi_{0, T} \mid \mathcal{F}_{t}\right), \quad 0 \leqslant t \leqslant T$. Thus, the forward price $F_{t}$ is given by

$$
\begin{aligned}
F_{t} & =E^{\widetilde{P}}\left(F_{T} \mid \mathcal{F}_{t}\right)=E\left(F_{T} \Phi_{t, T} \mid \mathcal{F}_{t}\right) \\
& =E\left(I_{T} \Phi_{t, T} \mid \mathcal{F}_{t}\right), \quad 0 \leqslant t \leqslant T .
\end{aligned}
$$

In general, the pricing kernel is characterized by the Girsanov-functional and thus it is not necessarily a deterministic function of $I_{t}$ or $F_{t}$. However, in a representative investor economy with a state-independent von NeumannMorgenstern utility function over the terminal asset price $F_{T}$ the pricing kernel is characterized by a deterministic function of time $t$ and either $I_{t}$ or $F_{t}$. This follows from the equilibrium condition

$$
\Phi_{0, T}=a \frac{\partial}{\partial x} U\left(F_{T}\right)
$$

with $a$ some positive scalar and $U$ the state-independent utility function of the representative investor. ${ }^{7}$ The pricing kernel considered in this article is consistent with a representative investor economy. Given the information process of equation (1) with $I_{T}=F_{T}$, the forward price $F_{t}$ can then be characterized by the following backward stochastic differential equation

$$
\begin{aligned}
d F_{t} & =\underbrace{\left\{\frac{\partial}{\partial t} v\left(t, I_{t}\right)+\frac{1}{2} \frac{\partial^{2}}{\partial x^{2}} v\left(t, I_{t}\right)\left(\sigma I_{t}\right)^{2}\right\}}_{=\mu_{t} F_{t}} d t+\underbrace{\frac{\partial}{\partial x} v\left(t, I_{t}\right) \sigma I_{t}}_{=\Sigma_{t} F_{t}} d W_{t}, \\
0 & \leqslant t \leqslant T, \\
v\left(T, I_{T}\right) & =I_{T},
\end{aligned}
$$

$$
{ }^{7} \text { For. }
$$

${ }^{7}$ For a more detailed discussion see for example Decamps and Lazrak [15] or Camara 
with $v\left(t, I_{t}\right)=F_{t}$ and the instantaneous drift $\mu_{t}$ and the instantaneous volatility $\Sigma_{t}$ being deterministic functions of time $t$ and $F_{t}$. Note that we can derive a similar stochastic differential equation for any state variable $X_{t}$, given that $F_{T}$ is a deterministic function of $X_{T}$.

\section{Asset returns in continuous-time}

\subsection{Properties of asset price processes for different char- acterizations of the pricing kernel}

In this section we analyze the properties of the stochastic differential equation (2) governing the forward price process. For an information process governed by equation (1) and a pricing kernel with constant elasticity it is known that the forward price is governed by a geometric Brownian motion. In this case the forward price is given by

$$
F_{t}^{\mathrm{C}}=v^{\mathrm{C}}\left(t, I_{t}\right)=A(t) I_{t}, \quad 0 \leqslant t \leqslant T,
$$

where $A(t)$ is a deterministic function of time $t$. Thus, in this case expected returns do not depend on the level of $I_{t}$ nor $F_{t}$. Moreover, the elasticity of the forward price with respect to $I_{t}\left(\eta_{t}^{F, I} \equiv \frac{\partial v\left(t, I_{t}\right)}{\partial x} \frac{I_{t}}{v\left(t, I_{t}\right)}\right)$ is equal to 1 and the instantaneous volatility $\Sigma_{t}$ of the forward price process $F$ is equal to the instantaneous volatility $\sigma$ of the information process $I$.

However, the following Lemma states that the elasticity of the forward price with respect to $I_{t}$ is higher [lower] than 1 for declining [increasing] elasticity of the pricing kernel. ${ }^{8}$ This result is closely related to Theorem 3 of Franke, Stapleton and Subrahmanyam [18] who show that the ratio between the forward price under declining elasticity of the pricing kernel and the forward price under constant elasticity of the pricing kernel increases with the level of the latter one.

Lemma 1 Assume that the information process is governed by a geometric Brownian motion with constant instantaneous volatility and no drift. Then

\footnotetext{
${ }^{8}$ In this paper we consider only monotonically declining [increasing] elasticity of the pricing kernel. The empirical evidence on the elasticity of the pricing kernel is mixed. However there are studies that report nonmonotonic elasticity of the pricing kernel, see Jackwerth [22].
} 
the elasticity of the forward price with respect to $I_{t}$ is higher [lower] than 1, i.e. $\frac{\partial v\left(t, I_{t}\right)}{\partial x} \frac{I_{t}}{v\left(t, I_{t}\right)}>[<] 1$, if and only if the elasticity of the pricing kernel is declining [increasing].

Proof. See Appendix A.1

Since $\eta_{t}^{F, I}>[<] 1$ implies that a $1 \%$ change in $I_{t}$ leads to a more [less] than $1 \%$ change in $F_{t}$, Lemma 1 establishes that the forward price overreacts [underreacts] compared to the case of constant elasticity of the pricing kernel if the elasticity of the pricing kernel is declining [increasing]. To get the intuition for the overreaction [underreaction] let us think about the elasticity of the pricing kernel in terms of relative risk aversion of the representative investor. A representative investor with decreasing [increasing] relative risk aversion requires a lower [higher] return for the same risk the wealthier he is. Compared to an investor with constant relative risk aversion, his required relative risk premium $\left(\frac{I_{t}-F_{t}}{F_{t}}\right)$ decreases [increases] the wealthier he is. Hence, the price he is willing to pay for the asset increases more [less] with increasing expected terminal value. Thus, with nonconstant relative risk aversion a change in the expected terminal value $I_{t}$ does also induce a change of the required risk premium. This change of the risk premium reinforces [diminishes] the purely 'information based' change of the asset price. Thus, since the required risk premium decreases [increases] with the level of $I_{t}$ for declining [increasing] elasticity of the pricing kernel the forward price overreacts [underreacts].

The following proposition establishes that predictability of asset returns and nonconstant elasticity of the pricing kernel are closely related. It is shown that predictability of asset returns is a consequence of the overreaction [underreaction] effect.

Proposition 1 Assume that the information process is governed by a geometric Brownian motion with constant instantaneous volatility and no drift. Then the expected return of the forward price process depends negatively [positively] on the level of the forward price, if and only if the elasticity of the pricing kernel is declining [increasing]. In addition, the instantaneous volatility of the forward price is higher [lower] than the instantaneous volatility of the information process, if and only if the elasticity of the pricing kernel is declining [increasing].

Proof. See Appendix A.2 
What do we learn from Proposition 1 and what is the economic mechanism which drives the results? First, the higher [lower] instantaneous volatility is a direct consequence of the overreaction [underreaction] effect. The instantaneous volatility of the forward price $\Sigma_{t}$ measures the instantaneous reaction of the forward price to innovations of the Brownian motion $W$. This Brownian motion drives both processes $I$ and $F$. Since $F$ overreacts [underreacts] relatively to $I, \Sigma_{t}$ must be higher [lower] than $\sigma$. This intuitive relation between $\Sigma_{t}$ and $\sigma$ is also seen from the definition of $\Sigma_{t}$

$$
\Sigma_{t}=\eta_{t}^{F, I} \sigma, \quad 0 \leqslant t \leqslant T .
$$

Second, expected returns depend negatively [positively] on the level of the forward price if the elasticity of the pricing kernel is declining [increasing]. Thus, we have mean reversion [mean aversion] in returns. This effect is also due to the changing risk premium. The higher $I_{t}$ the lower [higher] will be the risk premium under declining [increasing] elasticity. Therefore the expected return decreases [increases] with the level of $I_{t}$. The mean reversion [mean aversion] can also be related to the overreaction [underreaction] effect. Note first that the distribution of the terminal asset price is independent of the pricing kernel and equal to the distribution of $I_{T}$. However, under declining [increasing] elasticity of the pricing kernel the forward price overreacts [underreacts]. Hence, since $I_{T}=F_{T}$ this overreaction [underreaction] has to be compensated and thus asset returns exhibit mean reversion [mean aversion].

The overreaction effect also provides an additional intuition for the mispricing of the Black-Scholes model when the elasticity of the pricing kernel is not constant. This effect has been analyzed in detail by Franke, Stapleton and Subrahmanyam [18]. They show that options are more expensive with declining elasticity than with constant elasticity of the pricing kernel. In the light of our results this overpricing compared to Black-Scholes follows because of the higher instantaneous volatility of the forward price process. It is well known that hedging costs are increasing with the instantaneous volatility. Thus, although the terminal value is distributed as in the constant elasticity case, under declining elasticity hedging costs are higher. Higher hedging costs, however, imply a higher price. Hence, option prices exceed Black-Scholes prices when the pricing kernel has declining elasticity.

Let us now consider the characteristics of the instantaneous volatility in more detail. The following proposition provides a new explanation for the empirically well documented asymmetric volatility phenomenon. 
Proposition 2 Assume that the information process is governed by a geometric Brownian motion with constant instantaneous volatility and no drift. Then the instantaneous volatility of the forward price is decreasing [increasing] in the level of the forward price if the elasticity of the pricing kernel is declining [increasing].

\section{Proof. See Appendix A.3}

Combining this result with Proposition 1 gives a clear characteristic of the instantaneous volatility in the case of declining [increasing] elasticity of the pricing kernel. Note, however, that the relationship established in Proposition 2 is not necessarily monotonic. Let us now discuss this relationship for the case of declining elasticity: Why is the instantaneous volatility $\Sigma_{t}^{\mathrm{DE}}$ of the forward price process smaller when forward prices are high than when forward prices are low? This effect comes from the fact that we assume risk aversion over $\mathbb{R}^{+}$. Thus, for $I_{t} \rightarrow \infty$ the representative investor is risk neutral and therefore also constant relative risk averse.

This explanation for asymmetric volatility, i.e. high volatility in bear markets and low volatility in bull markets differs from the two previous approaches, i.e. the leverage effect (see for example Black [7] and Christie [13]) and the volatility feedback effect (see for example Campbell and Hentschel [11] and Lüders and Peisl [25]). Hence, Proposition 2 presents a third mechanism which might contribute to the observed asymmetric volatility. Moreover, recent empirical results show that the negative correlation between volatility and returns is more pronounced for market returns than for individual stocks. This, however, suggests that the leverage effect is less important than the preference based arguments, i.e. volatility feedback and declining elasticity. ${ }^{9}$

\subsection{Stationarity}

The purpose of the next section is to analyze the characteristics of the stochastic processes in terms of moments of discretely sampled asset returns as for example the autocorrelation and standard deviation of such returns. The following analysis, however, shows that usually asset returns are not stationary. Stationarity requires that the forward price process (the return process) is governed by a time-homogeneous stochastic differential equation, i.e. $\mu_{t}$

\footnotetext{
${ }^{9}$ See for example Andersen, Bollerslev, Diebold and Ebens [1], Hentschel [21], Mayhew and Stivers [26] and Tauchen, Zhang and Liu [28].
} 
and $\Sigma_{t}$ of the stochastic differential equation (2) for the price $F_{t}$ may depend on $F_{t}$, but they may not depend on time $t .^{10}$

Proposition 3 Assume that the information process is governed by a geometric Brownian motion with constant instantaneous volatility and no drift. Then the forward price process is governed by a time-homogeneous stochastic differential equation if and only if the elasticity of the pricing kernel is constant.

Proof. See Appendix A.4

While Proposition 3 does not really come as a surprise, it has strong implications for empirical studies of asset prices. The assumption of a lognormal distribution of the terminal value, time independent coefficients and nonconstant elasticity of the pricing kernel are incompatible. Moreover, note that since the forward price process is governed by a geometric Brownian motion if the elasticity of the pricing kernel is constant, it follows that either the forward price process is governed by a geometric Brownian motion or it is not governed by a time-homogeneous stochastic differential equation. The intuition behind this result is as follows. With, for example, declining elasticity of the pricing kernel the asset price instantaneously overreacts. This overreaction is then compensated by the mean reversion. However, both effects depend on the distance to the terminal date $T$ since at the terminal date the forward price is equal to the lognormally distributed $I_{T}$. The important point of Proposition 3 is that most estimation techniques rely on the assumption of time-homogeneity. ${ }^{11}$ Hence, empirical studies might suffer from the nonstationarity of asset returns.

\footnotetext{
${ }^{10}$ See also He and Leland [20] for the analysis of time-homogeneous asset price processes. For the estimation of diffusion models see for example Gourieroux and Jasiak [19]. A recent development on the estimation of diffusion processes is found, for example, in Elerian, Chib, Shephard [16].

${ }^{11}$ For an overview of recent estimation techniques see for example Gourieroux and Jasiak [19].
} 


\section{Implications for asset returns in discrete- time}

\subsection{Time series properties of asset returns}

Even though Proposition 3 suggests careful interpretation of empirical results we may deduce some properties of the return process over finite intervals. It is shown in Proposition 1 that for declining [increasing] elasticity of the pricing kernel the forward price is characterized by a function $v$ satisfying

$$
\begin{aligned}
& \frac{\partial}{\partial \ln x} \ln v^{\mathrm{DE}}\left(t, I_{t}\right)>1, \quad 0 \leqslant t<T, \\
& \frac{\partial}{\partial \ln x} \ln v^{\mathrm{IE}}\left(t, I_{t}\right)<1, \quad 0 \leqslant t<T .
\end{aligned}
$$

It follows immediately that the variance of $\ln v^{\mathrm{DE}}\left(t, I_{t}\right)$ is higher and the variance of $\ln v^{\mathrm{IE}}\left(t, I_{t}\right)$ is smaller than the variance of $\ln I_{t}$ for $0 \leqslant t<T$. For constant elasticity of the pricing kernel both variances are equal since in this case the elasticity of the forward price with respect to $I_{t}$ equals 1 . Note also that this is true for conditional as well as unconditional variances.

More relevant are the properties of the returns $r_{t, \tau}=\ln F_{\tau}-\ln F_{t}$. The following proposition shows that for the case of declining elasticity of the pricing kernel, the conditional variance of returns over finite periods, $\operatorname{Var}\left(\ln F_{\tau}-\ln F_{t} \mid \mathcal{F}_{t}\right)$, and the unconditional variance of returns over finite periods, $\operatorname{Var}\left(\ln F_{\tau}-\ln F_{t}\right)$, are higher than under constant elasticity of the pricing kernel. Moreover, it should be noted that the results are not sensitive to whether we consider $\operatorname{Var}\left(\ln F_{\tau}-\ln F_{t}\right)$ or $\operatorname{Var}\left(\ln F_{\tau}-\ln F_{t} \mid \mathcal{F}_{t-\theta}\right)$ with $\theta>0$. Important is only whether $\ln F_{t}$ is measurable with respect to the filtration on which the variance is conditioned.

Proposition 4 Suppose that the information process is governed by a geometric Brownian motion with constant instantaneous volatility and no drift. Then the conditional and unconditional variance of returns over finite periods $\left(\ln F_{\tau}-\ln F_{t}\right.$ with $\left.0 \leq t<\tau<T\right)$ is higher than the corresponding variance of the returns of the information process $\left(\ln I_{\tau}-\ln I_{t}\right.$ with $\left.0 \leq t<\tau<T\right)$ when the elasticity of the pricing kernel is declining.

Proof. See Appendix A.5 
In addition, note that the conditional and unconditional variance of returns over finite periods is equal to the corresponding variance of the information process if the elasticity is constant. However, while the conditional variance $\operatorname{Var}\left(\ln F_{\tau}-\ln F_{t} \mid \mathcal{F}_{t}\right)$ is lower for increasing elasticity of the pricing kernel, this is not necessarily true for $\operatorname{Var}\left(\ln F_{\tau}-\ln F_{t} \mid \mathcal{F}_{t-\theta}\right)$ with $\theta>0$. To see this, consider

$$
\begin{aligned}
\operatorname{Var}\left(\ln F_{\tau}-\ln F_{t} \mid \mathcal{F}_{t-\theta}\right)= & \operatorname{Var}\left(E\left(\ln F_{\tau} \mid \mathcal{F}_{t}\right)-\ln F_{t} \mid \mathcal{F}_{t-\theta}\right) \\
& +E\left(\operatorname{Var}\left(\ln F_{\tau} \mid \mathcal{F}_{t}\right) \mid \mathcal{F}_{t-\theta}\right)
\end{aligned}
$$

with $E\left(\operatorname{Var}\left(\ln F_{\tau} \mid \mathcal{F}_{t}\right) \mid \mathcal{F}_{t-\theta}\right)$ being lower under increasing elasticity than under constant elasticity of the pricing kernel. However, while in the case of constant elasticity of the pricing kernel $\operatorname{Var}\left(E\left(\ln F_{\tau} \mid \mathcal{F}_{t}\right)-\ln F_{t} \mid \mathcal{F}_{t-\theta}\right)=0$ this is positive for nonconstant elasticity. Hence, in contrast to the case of declining elasticity the effect on the unconditional variance is ambiguous under increasing elasticity, since the first term on the right hand side is higher than under constant elasticity but the second term is lower.

The intuition for the higher variance of returns when the pricing kernel has declining elasticity is the same as for the instantaneous volatility. The change in the risk premium increases the reaction to a change in expectations compared to the case of constant elasticity of the pricing kernel. This leads to a higher variance of returns. Moreover, Proposition 4 is closely related to Proposition 3 since it illustrates the nonstationarity of asset returns. For declining elasticity of the pricing kernel the conditional variance of returns over any subperiod $[t, t+\theta]$ with $0 \leq t<t+\theta<T$, $\operatorname{Var}\left(\ln F_{t+\theta}-\ln F_{t} \mid \mathcal{F}_{t}\right)$, is higher than the corresponding variance of the information process $\operatorname{Var}\left(\ln I_{t+\theta}-\ln I_{t} \mid \mathcal{F}_{t}\right)$ except for the returns over the period $[T-\theta, T]$. The conditional variance of terminal returns $r_{t, T}$ is always equal to the corresponding variance of the information process, i.e.

$$
\operatorname{Var}\left(\ln F_{T}-\ln F_{t} \mid \mathcal{F}_{t}\right)=\operatorname{Var}\left(\ln I_{T}-\ln I_{t} \mid \mathcal{F}_{t}\right), \quad 0 \leqslant t \leqslant T .
$$

In contrast, the corresponding conditional variance of the information process is equal for both periods, i.e.

$$
\operatorname{Var}\left(\ln I_{t+\theta}-\ln I_{t} \mid \mathcal{F}_{t}\right)=\sigma^{2} \theta, \quad 0 \leqslant t \leqslant t+\theta \leqslant T,
$$

which demonstrates that the transition density of asset returns depends on time $t$. Proposition 4 is also in line with Franke, Stapleton and Subrahmanyam [18] who have shown that the variance of the forward price is higher under the declining elasticity pricing kernel. 
In the previous section we derived the fact that returns are predictable under a pricing kernel with nonconstant elasticity. The following proposition shows that the terminal return $r_{\tau, T}$ is conditionally negatively [positively] correlated with the preceding return $r_{t, \tau}$ with $0 \leq t<\tau<T$ if the elasticity of the pricing kernel is declining [increasing].

Proposition 5 Suppose that the information process is governed by a geometric Brownian motion with constant instantaneous volatility and no drift. Then final period returns $\left(r_{\tau, T}=\ln F_{T}-\ln F_{\tau}\right.$ with $\left.0<\tau<T\right)$ are conditionally negatively [positively] correlated with preceding returns $\left(r_{t, \tau}=\ln F_{\tau}-\ln F_{t}\right.$ with $0 \leq t<\tau<T)$, i.e. $\operatorname{Corr}\left(r_{\tau, T}, r_{t, \tau} \mid \mathcal{F}_{t}\right)<[>] 0$, if the elasticity of the pricing kernel is declining [increasing].

Proof. See Appendix A.6

Proposition 5 shows that in contrast to the case of constant elasticity of the pricing kernel, asset returns are negatively [positively] autocorrelated if the elasticity of the pricing kernel is declining [increasing]. Since Proposition 5 and Proposition 1 are more or less two views on the same mechanism, the economic intuition for the serial correlation parallels the explanation of Proposition 1. For declining elasticity, we have seen that asset prices overreact compared to the information process. Hence when, for example, expectations rise the asset price rises more than the change in expectations. Since $F_{T}=I_{T}$ this relatively high first period return has to be compensated by a relatively low return in the following period. In the case of increasing elasticity of the pricing kernel, asset prices underreact and since $F_{T}=I_{T}$ they have to catch up in the second period, which yields positive autocorrelation.

Finally, let us consider the variance ratio

$$
\nu r=\left(\frac{\tau-t}{T-t}\right) \frac{\operatorname{Var}\left(\ln F_{T}-\ln F_{t} \mid \mathcal{F}_{t}\right)}{\operatorname{Var}\left(\ln F_{\tau}-\ln F_{t} \mid \mathcal{F}_{t}\right)}, \quad 0<t<\tau<T,
$$

which is widely used in empirical finance to detect serial correlation. Since

$$
\operatorname{Var}\left(\ln F_{T} \mid \mathcal{F}_{t}\right)=\operatorname{Var}\left(\ln I_{T} \mid \mathcal{F}_{t}\right)
$$

and

$$
\operatorname{Var}\left(\ln F_{\tau}-\ln F_{t} \mid \mathcal{F}_{t}\right)>[<] \operatorname{Var}\left(\ln I_{\tau}-\ln I_{t} \mid \mathcal{F}_{t}\right)
$$

for declining [increasing] elasticity of the pricing kernel it is easily seen that the variance ratio is smaller [higher] than 1 for declining [increasing] elasticity of the pricing kernel which is consistent with our earlier finding of mean 
reversion and negative autocorrelation [mean aversion and positive autocorrelation]. Hence, in spite of the concerns expressed earlier given our assumptions the variance ratio test may be an appropriate technique to recover some characteristics of the return process.

\subsection{The explanatory power of multiples}

Up to here we have focused on univariate properties of the asset price process. However, there exist many other forms of predictability. This section analyzes the relationship between expected returns and multiples to understand why multiples may have predictive power although the market is efficient and in equilibrium. To analyze this question we slightly extend our previous model by introducing an additional process which describes the evolution of the book value of equity. More precisely, we assume that the book value of equity $b v_{t}$ is characterized by

$$
\begin{aligned}
& b v_{t}=b v_{0}+\int_{0}^{t} \kappa b v_{s} d s+\int_{0}^{t} \gamma b v_{s} d W_{s}, \quad 0 \leq t \leq T \\
& b v_{0}>0
\end{aligned}
$$

where we assume that $\kappa$ and $\gamma$ are some positive constant parameters. Thus, we assume that the book value of equity is governed by a geometric Brownian motion with constant coefficients. The book value of equity is only loosely related to the market value of equity since accounting numbers are distorted by regulations and do not necessarily reflect the fair value. However, it seems reasonable to assume that the book value of equity at time $T$ is equal to the value of the company at time $T$ when the company is liquidated. Hence, at time $T$ we have $b v_{T}=F_{T}$. The following derivation of the information process is based on the assumption that the representative investor observes the book value of equity and based on these observation he builds his conditional expectations about the terminal value $F_{T}$. With our assumptions the derivation of the information process $I_{t}=E\left(b v_{T} \mid \mathcal{F}_{t}\right)$ is straightforward.

Lemma 2 Assume that the book value of equity bv $v_{t}$ is governed by the geometric Brownian motion (4) and that at terminal date $T$ the market value of equity is equal to the book value of equity, i.e. $b v_{T}=F_{T}$. Then the information process is governed by a geometric Brownian motion with constant instantaneous volatility and no drift. Moreover, the instantaneous volatility of the information process is equal to the instantaneous volatility of the stochastic process governing the book value of equity. 


\section{Proof. See Appendix A.7}

Hence, as in the previous section the information process is governed by a geometric Brownian motion with constant instantaneous volatility and drift zero. Therefore our previous results can be applied to our extension of the model.

Proposition 6 Assume that the book value of equity bv $v_{t}$ is governed by the geometric Brownian motion (4) and that at terminal date $T$ the market value of equity is equal to the book value of equity, i.e. $b v_{T}=F_{T}$. Then, for declining and for increasing elasticity of the pricing kernel, the expected return depends positively on the book-to-market ratio $\left(\frac{b v_{t}}{F_{t}}\right)$.

Proof. See Appendix A.8

This result is a direct consequence of the derived mean reversion [mean aversion] of asset returns for declining [increasing] elasticity of the pricing kernel. A higher book-to-market ratio indicates that the risk premium is relatively high. Thus, the expected return is higher for high book-to-market ratios. For declining elasticity of the pricing kernel, the book-to-market ratio decreases with increasing $I_{t}$ while for increasing elasticity of the pricing kernel the book-to-market ratio increases with increasing $I_{t}$.

To analyze the predictive power of the price-earnings ratio $\left(\frac{F_{t}}{e_{t}}\right)$ we have to define earnings $\left(e_{t}\right)$ in terms of our model for the book value of equity. More precisely, we assume that the following relationship holds: ${ }^{12}$

$$
b v_{t}=b v_{t-1}+e_{t}, \quad 0 \leq t \leq T .
$$

Proposition 7 Assume that the book value of equity bv $v_{t}$ is governed by the geometric Brownian motion (4) and that at terminal date $T$ the market value of equity is equal to the book value of equity, i.e. $b v_{T}=F_{T}$. Moreover, consider the cases when earnings are positive, i.e. $e_{t}>0$. Then, conditionally on $b v_{t-1}$ the expected return depends negatively on the price-earnings ratio $\left(\frac{F_{t}}{e_{t}}\right)$ when the elasticity of the pricing kernel is increasing. For declining elasticity of the pricing kernel and conditionally on $b v_{t-1}$ expected returns depend negatively on the price-earnings ratio if

$$
e_{t}<\frac{\partial}{\partial x} B\left(t, I_{t}\right) \frac{v^{D E}\left(t, I_{t}\right)}{\frac{\partial}{\partial x} v^{D E}\left(t, I_{t}\right)}, \quad 0 \leq t \leq T,
$$

\footnotetext{
${ }^{12}$ Note that we define periods such that their length is 1 . This simplifies the notation. We assume that $T \gg 1$.
} 
with $b v_{t}=B\left(t, I_{t}\right)$ and $F_{t}=v^{D E}\left(t, I_{t}\right)$. For

$$
e_{t}>\frac{\partial}{\partial x} B\left(t, I_{t}\right) \frac{v^{D E}\left(t, I_{t}\right)}{\frac{\partial}{\partial x} v^{D E}\left(t, I_{t}\right)}, \quad 0 \leq t \leq T,
$$

expected returns depend positively on the price-earnings ratio.

Proof. See Appendix A.9

The explanatory power of the price-earnings ratio is also due to the fact that asset returns are mean reverting [mean averting] for declining [increasing] elasticity of the pricing kernel. However, Proposition 7 establishes that while the relationship is unequivocal for increasing elasticity of the pricing kernel, in the case of declining elasticity expected returns depend positively [negatively] on the price-earnings ratio if earnings are relatively high [low]. Why do we get this surprising result for declining elasticity of the pricing kernel? Assume for a moment $F_{t}$ constant. In this case obviously higher earnings would imply higher expected returns since the expected terminal value $I_{t}$ increases also. Moreover, price-earnings ratios would decrease also for $F_{t}$ fixed and increasing earnings. However, the forward price $F_{t}$ depends also positively on earnings. Since with declining elasticity of the pricing kernel the forward price may react heavily to a change in expectations and hence to earnings, this second effect may dominate the first. Thus, the total effect is ambiguous and depends on how strongly the forward price reacts to earnings.

\section{Conclusion}

Predictability of asset returns is often explained by nonrational expectations or new behavioral postulates which are not consistent with von NeumannMorgenstern utility functions. However, still little is known on return characteristics in a traditional framework when the elasticity of the pricing kernel is not constant. In this paper we derived the characteristics of asset returns when the elasticity of the pricing kernel is not constant. However, we assume rational expectations and the framework is also consistent with a representative investor with a von Neumann-Morgenstern utility function.

We show that with nonconstant elasticity of the pricing kernel, the forward price $F_{t}$ is not a linear function of the expected terminal value $I_{t}=$ $E\left(F_{T} \mid \mathcal{F}_{t}\right)$. This nonlinearity induces predictability in asset returns. The 
predictability is reflected by the fact that expected returns depend on the level of the asset price. We find also that financial ratios (as the book-tomarket ratio) have explanatory power for expected returns. While expected returns are always positively related to the book-to-market ratio the relationship between expected returns and price-earnings ratios is more complicated. For increasing elasticity of the pricing kernel there is always a negative relation but for declining elasticity the relationship between price-earnings ratios and expected returns is positive for relatively high earnings while it is negative for relatively low earnings. Moreover, nonconstant elasticity of the pricing kernel leads to time-varying instantaneous volatility. For declining [increasing] elasticity of the pricing kernel, the instantaneous volatility decreases [increases] with rising asset prices. Hence, declining elasticity of the pricing kernel leads to asymmetric volatility. In addition, we show that asset returns 'overreact' ['underreact'] to changes in expectations when the pricing kernel has declining [increasing] elasticity. We also analyzed the moments of the discretely sampled return process. The results suggest very careful interpretation of empirical results since the derived return processes are usually not stationary. In spite of this result, it is shown that declining [increasing] elasticity of the pricing kernel leads to negative [positive] autocorrelation of asset returns. Hence, the analysis shows that many return characteristics are compatible with a rational expectations model with nonconstant elasticity of the pricing kernel.

\section{A Appendix}

\section{A.1 Proof of Lemma 1}

The ratio between the forward price under declining [increasing] elasticity of the pricing kernel $F_{t}^{\mathrm{DE}}\left[F_{t}^{\mathrm{IE}}\right]$ and the forward price under constant elasticity $F_{t}^{\mathrm{C}}$

$$
\frac{F_{t}^{\mathrm{DE}}}{F_{t}^{\mathrm{C}}}, \text { respectively } \frac{F_{t}^{\mathrm{IE}}}{F_{t}^{\mathrm{C}}}
$$

increases [decreases] monotonically with increasing $F_{t}^{\mathrm{C}}$. This follows because $\frac{I_{t}}{F_{t}^{C}} \equiv \frac{E\left(F_{T} \mid \mathcal{F}_{t}\right)}{F_{t}^{C}}$ is independent of the level of $I_{t}$ since

$$
F_{t}^{\mathrm{C}}=v^{\mathrm{C}}\left(t, I_{t}\right)=A(t) I_{t}, \quad 0 \leqslant t \leqslant T
$$


with $A:[0, T] \rightarrow \mathbb{R}^{+}$, and with declining [increasing] elasticity of the pricing kernel the expected return $\frac{I_{t}}{F_{t}^{\mathrm{DE}}}\left(\frac{I_{t}}{F_{t}^{\mathrm{IE}}}\right)$ decreases [increases] with increasing $I_{t}$. For a more detailed proof see Theorem 3 in Franke, Stapleton and Subrahmanyam [18]. However, this statement in Franke, Stapleton and Subrahmanyam [18] can be expressed more formally as:

$$
\frac{\partial}{\partial x}\left(\frac{v^{\mathrm{IE}}\left(t, I_{t}\right)}{v^{\mathrm{C}}\left(t, I_{t}\right)}\right)<0, \quad 0 \leqslant t<T
$$

respectively

$$
\frac{\partial}{\partial x}\left(\frac{v^{\mathrm{DE}}\left(t, I_{t}\right)}{v^{\mathrm{C}}\left(t, I_{t}\right)}\right)>0, \quad 0 \leqslant t<T
$$

Simple calculus shows that this is equivalent to

$$
\frac{\partial v^{\mathrm{IE}}\left(t, I_{t}\right)}{\partial x}<\frac{v^{\mathrm{IE}}\left(t, I_{t}\right)}{I_{t}}, \quad 0 \leqslant t<T
$$

respectively

$$
\frac{\partial v^{\mathrm{DE}}\left(t, I_{t}\right)}{\partial x}>\frac{v^{\mathrm{DE}}\left(t, I_{t}\right)}{I_{t}}, \quad 0 \leqslant t<T
$$

\section{A.2 Proof of Proposition 1}

$\frac{I_{t}}{F_{t}}$ is characterized by a function $\psi:[0, T] \times \mathbb{R}^{+} \rightarrow \mathbb{R}^{+},(t, x) \rightarrow \frac{x}{v(t, x)}$ which does not depend on $I_{t}$ if and only if $\frac{\partial}{\partial x} v\left(t, I_{t}\right)$ depends only on time $t$, i.e. the forward price at time $t$ is linear in $I_{t}$. Positive [negative] dependence of the expected return implies

$$
\frac{\partial}{\partial x}\left(\frac{x}{v(t, x)}\right)>[<] 0
$$

Comparing equation (7) to equation (5) and equation (6) proves that declining [increasing] elasticity of the pricing kernel yields negative [positive] dependence of the expected return on the level of the forward price. Since $v(t, x)$ and $x$ are strictly positive, the results hold also for the expected return $\ln \left(\frac{I_{t}}{F_{t}}\right)$. That the instantaneous volatility $\Sigma_{t}$ of the forward price process $F$ is higher [lower] under declining [increasing] elasticity of the pricing kernel 
follows from Lemma 1 and the definition of the instantaneous volatility of the forward price process

$$
\Sigma_{t}=\frac{\frac{\partial}{\partial x} v\left(t, I_{t}\right)}{v\left(t, I_{t}\right)} \sigma I_{t}, \quad 0 \leqslant t \leqslant T
$$

\section{A.3 Proof of Proposition 2}

We give the proof for declining elasticity, the proof for increasing elasticity is analogous. Since $\frac{\partial}{\partial x}\left(\frac{v^{\mathrm{DE}}\left(t, I_{t}\right)}{I_{t}}\right) \geq 0$ and $\frac{{ }^{\mathrm{DE}}\left(t, I_{t}\right)}{I_{t}} \leq 1$ for $0 \leq t<T$ it follows from the Theorem of Bolzano-Weierstrass that

$$
\lim _{I_{t} \rightarrow \infty}\left(\frac{v^{\mathrm{DE}}\left(t, I_{t}\right)}{I_{t}}\right)=c
$$

where $c$ is some positive constant with $c \leq 1$ and $F_{t}=v^{\mathrm{DE}}\left(t, I_{t}\right)$. Since $\lim _{I_{t} \rightarrow \infty} v^{\mathrm{DE}}\left(t, I_{t}\right)=\infty$ it follows from the rule of L'Hopital that

$$
c=\lim _{I_{t} \rightarrow \infty}\left(\frac{v^{\mathrm{DE}}\left(t, I_{t}\right)}{I_{t}}\right)=\lim _{I_{t} \rightarrow \infty}\left(\frac{\partial}{\partial x} v^{\mathrm{DE}}\left(t, I_{t}\right)\right) .
$$

Hence, the elasticity of the forward price with respect to $I_{t}$ converges to 1 , i.e.

$$
\lim _{I_{t} \rightarrow \infty}\left(\frac{\partial}{\partial x} v^{\mathrm{DE}}\left(t, I_{t}\right) \frac{I_{t}}{v^{\mathrm{DE}}\left(t, I_{t}\right)}\right)=1 .
$$

For $I_{t}<\infty$ we have already seen that the elasticity is higher than 1 . Hence,

$$
\lim _{I_{t} \rightarrow \infty}\left(\Sigma_{t}^{\mathrm{DE}}\right)=\sigma
$$

while $\Sigma_{t}^{\mathrm{DE}}>\sigma \forall I_{t}<\infty$.

\section{A.4 Proof of Proposition 3}

First note that because $\frac{\partial v\left(T, I_{T}\right)}{\partial x} \frac{I_{T}}{v\left(T, I_{T}\right)}=1$ and $\Sigma_{t}=\frac{\partial v\left(t, I_{t}\right)}{\partial x} \frac{I_{t}}{v\left(t, I_{t}\right)} \sigma, \quad 0 \leqslant t \leqslant$ $T$ we know that the instantaneous volatility $\Sigma_{t}$ is constant in $T$ with $\Sigma_{T}=\sigma$. Moreover, the instantaneous volatility $\Sigma_{t}$ can be characterized by a function 
$\widetilde{\Sigma}\left(t, F_{t}\right)=\Sigma_{t}$. Hence, we have $\widetilde{\Sigma}(T, x)=$ constant for all $x$. If the elasticity of the pricing kernel is declining [increasing] then

$$
\widetilde{\Sigma}\left(t, F_{t}\right)>[<] \sigma, \quad 0 \leqslant t<T,
$$

for all finite values of $F_{t}$. Since $\widetilde{\Sigma}\left(T, F_{T}\right)=\sigma$, the instantaneous volatility of the forward price depends on time $t$. It follows from Proposition 1 that $\widetilde{\Sigma}\left(t, F_{t}\right)=\sigma$ if and only if the elasticity of the pricing kernel is constant.

\section{A.5 Proof of Proposition 4}

Since with declining elasticity of the pricing kernel $\operatorname{Var}\left(\ln F_{\tau} \mid \mathcal{F}_{t}\right)>\operatorname{Var}\left(\ln I_{\tau} \mid \mathcal{F}_{t}\right)$ for $0 \leq t<\tau<T$, it follows immediately that also

$$
\operatorname{Var}\left(\ln F_{\tau}-\ln F_{t} \mid \mathcal{F}_{t}\right)>\operatorname{Var}\left(\ln I_{\tau}-\ln I_{t} \mid \mathcal{F}_{t}\right), \quad 0 \leq t<\tau<T .
$$

Hence, the conditional variance of returns is higher under declining elasticity of the pricing kernel. Consider now the unconditional variance:

$$
\begin{aligned}
\operatorname{Var}\left(\ln F_{\tau}-\ln F_{t}\right) & =\operatorname{Var}\left(E\left(\ln F_{\tau} \mid \mathcal{F}_{t}\right)-\ln F_{t}\right)+E\left(\operatorname{Var}\left(\ln F_{\tau} \mid \mathcal{F}_{t}\right)\right) \\
0 & \leqslant t \leqslant \tau \leqslant T
\end{aligned}
$$

with

$$
E\left(\ln F_{\tau} \mid \mathcal{F}_{t}\right)-\ln F_{t}=E\left(\int_{t}^{\tau} \mu-\frac{1}{2} \Sigma^{2} d s \mid \mathcal{F}_{t}\right), \quad 0 \leqslant t \leqslant \tau \leqslant T .
$$

Equation (9) shows that except for constant elasticity of the pricing kernel with nonrandom term $\mu-\frac{1}{2} \Sigma^{2}$

$$
\operatorname{Var}\left(E\left(\ln F_{\tau} \mid \mathcal{F}_{t}\right)-\ln F_{t}\right)>0 .
$$

Moreover,

$$
\begin{aligned}
\operatorname{Var}\left(\ln I_{\tau}-\ln I_{t}\right) & =\operatorname{Var}\left(\ln I_{\tau} \mid \mathcal{F}_{t}\right) \\
0 & \leqslant t \leqslant \tau \leqslant T
\end{aligned}
$$

since the information process is governed by a geometric Brownian motion with constant volatility and no drift. Thus, because $\operatorname{Var}\left(\ln F_{\tau} \mid \mathcal{F}_{t}\right)>$ $\operatorname{Var}\left(\ln I_{\tau} \mid \mathcal{F}_{t}\right)$, we have

$$
\operatorname{Var}\left(\ln F_{\tau}-\ln F_{t}\right)>\operatorname{Var}\left(\ln I_{\tau}-\ln I_{t}\right) .
$$




\section{A.6 Proof of Proposition 5}

Since $\operatorname{sgn} \operatorname{Cov}\left(r_{\tau, T}, r_{t, \tau} \mid \mathcal{F}_{t}\right)=\operatorname{sgn} \operatorname{Cov}\left(\frac{E\left(F_{T} \mid \mathcal{F}_{\tau}\right)}{F_{\tau}}, F_{\tau} \mid \mathcal{F}_{t}\right)$ we have to consider

$$
\frac{\partial}{\partial x}\left(\frac{I\left(t, F_{t}\right)}{F_{t}}\right)
$$

where $I\left(t, F_{t}\right)=E\left(F_{T} \mid \mathcal{F}_{\tau}\right)$ is the inverse function of $F\left(t, I_{t}\right)$. Note that $F\left(t, I_{t}\right)$ is monotone in $I_{t}$. Hence, we have negative [positive] conditional autocorrelation for

which is equivalent to

$$
\frac{\partial}{\partial x} I\left(t, F_{t}\right)<[>] \frac{I\left(t, F_{t}\right)}{F_{t}}
$$

$$
\frac{\partial}{\partial x} F\left(t, I_{t}\right)<[>] \frac{F_{t}}{I\left(t, F_{t}\right)}
$$

It follows from Proposition 1 and Appendix A.2 that returns are conditionally negatively [positively] autocorrelated for declining [increasing] elasticity of the pricing kernel.

\section{A.7 Proof of Lemma 2}

Since $b v_{T}=F_{T}=I_{T}$, it follows from the Theorem of Feynman-Kac that $I_{t}$ is characterized by a function $\chi:[0, T] \times \mathbb{R} \rightarrow \mathbb{R}$ with $\chi\left(t, b v_{t}\right)=I_{t}$ for all $0 \leq t \leq T$, and $\chi(t, x)$ satisfies the following deterministic partial differential equation

$$
\begin{aligned}
0 & =\frac{\partial}{\partial t} \chi(t, x)+\frac{\partial}{\partial x} \chi(t, x) \kappa x+\frac{1}{2} \frac{\partial^{2}}{\partial x^{2}} \chi(t, x) \gamma^{2} x^{2}, \\
0 & \leqslant t \leqslant T, \\
\chi(T, x) & =x .
\end{aligned}
$$

Solving equation (10) and applying Ito's Lemma yields the following stochastic differential equation for the information process $I$ :

$$
\begin{aligned}
d I_{t} & =I_{t} \gamma d W_{t}, \quad 0 \leq t \leq T, \\
I_{0} & =E\left(b v_{T}\right) .
\end{aligned}
$$

Hence, the information process is governed by a geometric Brownian motion with constant instantaneous volatility and drift zero. The instantaneous volatility of the information process equals the instantaneous volatility of the book value of equity. 


\section{A.8 Proof of Proposition 6}

Note that $I_{t}=\chi\left(t, b v_{t}\right)$ is a deterministic function of the book value:

$$
I_{t}=\chi\left(t, b v_{t}\right)=\exp (\kappa(T-t)) b v_{t}, \quad 0 \leqslant t \leqslant T .
$$

It follows from equation (11) that we can consider $b v_{t}$ as a deterministic function $B$ of $I_{t}, B:[0, T] \times \mathbb{R}^{+} \rightarrow \mathbb{R}^{+}$:

$$
B=I_{t} \exp (-\kappa(T-t)), \quad 0 \leqslant t \leqslant T
$$

Hence, the book-to-market ratio is characterized by $\frac{B\left(t, I_{t}\right)}{v^{\mathrm{DE}}\left(t, I_{t}\right)}$ and for declining [increasing] elasticity of the pricing kernel

$$
\begin{aligned}
\frac{\partial}{\partial x}\left(\frac{B\left(t, I_{t}\right)}{v^{\mathrm{DE}}\left(t, I_{t}\right)}\right) & =\frac{\exp (-\kappa(T-t))}{\left(v^{\mathrm{DE}}\left(t, I_{t}\right)\right)}\left(1-\frac{\frac{\partial}{\partial x}\left(v^{\mathrm{DE}}\left(t, I_{t}\right)\right) I_{t}}{v^{\mathrm{DE}}\left(t, I_{t}\right)}\right)<[>] 0, \\
0 & \leqslant t<T,
\end{aligned}
$$

since the elasticity of the forward price is higher [lower] than 1 under the declining [increasing] elasticity pricing kernel. It follows from Proposition 1 that the expected return depends negatively [positively] on $I_{t}$ for declining [increasing] elasticity of the pricing kernel. We can conclude that the expected return depends positively on the book-to-market ratio.

\section{A.9 Proof of Proposition 7}

Let us first discuss the case of increasing elasticity of the pricing kernel. Consider therefore the first derivative of the price-earnings ratio with respect to the information process, i.e.

$$
\frac{\partial}{\partial x}\left(\frac{v^{\mathrm{IE}}\left(t, I_{t}\right)}{B\left(t, I_{t}\right)-B_{t-1}}\right), \quad 0 \leqslant t<T,
$$

with $B\left(t-1, I_{t-1}\right):=B_{t-1}=$ constant. Equation (12) may be rewritten as

$$
\frac{\frac{\partial}{\partial x} v^{\mathrm{IE}}\left(t, I_{t}\right)\left(B\left(t, I_{t}\right)-B_{t-1}\right)-\frac{\partial}{\partial x} B\left(t, I_{t}\right) v^{\mathrm{IE}}\left(t, I_{t}\right)}{\left(B\left(t, I_{t}\right)-B_{t-1}\right)^{2}}, \quad 0 \leqslant t \leqslant T .
$$

For positive earnings, i.e. $B\left(t, I_{t}\right)-B_{t-1}>0$, this term is negative, if

$$
\frac{\partial}{\partial x} v^{\mathrm{IE}}\left(t, I_{t}\right) \frac{I_{t}}{v^{\mathrm{IE}}\left(t, I_{t}\right)}<\frac{\partial}{\partial x} B\left(t, I_{t}\right) \frac{I_{t}}{B\left(t, I_{t}\right)-B_{t-1}}, \quad 0 \leqslant t \leqslant T .
$$


Since $\frac{\partial}{\partial x} B\left(t, I_{t}\right) \frac{I_{t}}{B\left(t, I_{t}\right)}=1$ (see, Lemma 1) and for increasing elasticity of the pricing kernel

$$
\frac{\partial v^{\mathrm{IE}}\left(t, I_{t}\right)}{\partial x} \frac{I_{t}}{v^{\mathrm{IE}}\left(t, I_{t}\right)}<1, \quad 0 \leqslant t<T
$$

(see, Proposition 1) it follows that the price-earnings ratio depends negatively on $I_{t}$. Since expected returns depend positively on $I_{t}$ (see, Proposition 1) we conclude that expected returns and price-earnings ratios are negatively related.

For declining elasticity of the pricing kernel, the price earnings ratio depends positively on $I_{t}$ if

$$
\frac{\partial}{\partial x} v^{\mathrm{DE}}\left(t, I_{t}\right) \frac{I_{t}}{v^{\mathrm{DE}}\left(t, I_{t}\right)}>\frac{\partial}{\partial x} B\left(t, I_{t}\right) \frac{I_{t}}{B\left(t, I_{t}\right)-B_{t-1}}, \quad 0 \leqslant t \leqslant T .
$$

However, for increasing elasticity of the pricing kernel

$$
\frac{\partial v^{\mathrm{DE}}\left(t, I_{t}\right)}{\partial x} \frac{I_{t}}{v^{\mathrm{DE}}\left(t, I_{t}\right)}>1, \quad 0 \leqslant t<T
$$

and therefore

$$
\frac{\partial}{\partial x}\left(\frac{v^{\mathrm{DE}}\left(t, I_{t}\right)}{B\left(t, I_{t}\right)-B_{t-1}}\right) \gtrless 0, \quad 0 \leqslant t<T,
$$

if

$$
e_{t} \gtrless \frac{\partial}{\partial x} B\left(t, I_{t}\right) \frac{v^{\mathrm{DE}}\left(t, I_{t}\right)}{\frac{\partial}{\partial x} v^{\mathrm{DE}}\left(t, I_{t}\right)}, \quad 0 \leq t \leq T .
$$

Since, expected returns depend negatively on $I_{t}$ we conclude that expected returns and price-earnings ratios are positively related for relatively low earnings, i.e.

$$
e_{t}<\frac{\partial}{\partial x} B\left(t, I_{t}\right) \frac{v^{\mathrm{DE}}\left(t, I_{t}\right)}{\frac{\partial}{\partial x} v^{\mathrm{DE}}\left(t, I_{t}\right)}, \quad 0 \leq t \leq T .
$$

and negatively related for relatively high earnings, i.e.

$$
e_{t}>\frac{\partial}{\partial x} B\left(t, I_{t}\right) \frac{v^{\mathrm{DE}}\left(t, I_{t}\right)}{\frac{\partial}{\partial x} v^{\mathrm{DE}}\left(t, I_{t}\right)}, \quad 0 \leq t \leq T .
$$




\section{References}

[1] Andersen, T. G., Bollerslev, T., Diebold, F. X. and Ebens H. (2001). The distribution of realized stock return volatility, Journal of Financial Economics, 61, 43-76.

[2] Barberis, N. and Huang, M. (2001). Mental Accounting, Loss Aversion, and Individual Stock Returns, Journal of Finance, 56, 1247-1292.

[3] Barberis, N., Huang, M. and Santos, T. (2001). Prospect Theory and Asset Prices, Quarterly Journal of Economics, 116, 1-53.

[4] Barberis, N., Shleifer, A. and Vishny, R. (1998). A Model of Investor Sentiment, Journal of Financial Economics, 49, 307-343.

[5] Bick, A. (1987). On the Consistency of the Black-Scholes Model with a General Equilibrium Framework, Journal of Financial and Quantitative Analysis 22, 259-275.

[6] Bick, A. (1990). On Viable Diffusion Price Processes of the Market Portfolio, Journal of Finance 45, 673-689.

[7] Black, F. (1976). Studies of Stock Market Volatility Changes. Proceedings of the American Statistical Association, Business and Economic Statistics Section, 177-181.

[8] Black, F. and Scholes, M. (1973). The Pricing of Options and Corporate Liabilities, Journal of Political Economy, 81, 637-654.

[9] Brennan, M. J. (2001). Mental Accounting, Loss Aversion, and Individual Stock Returns - Discussion, Journal of Finance, 56, 1292-1295.

[10] Camara, A. (2001). Option Prices Sustained by Risk-Preferences, Working Paper, University of Strathclyde.

[11] Campbell, J. Y. and Hentschel, L. (1992). No News is Good News: An Asymmetric Model of Changing Volatility in Stock Returns, Journal of Financial Economics, 31, 281-318.

[12] Cochrane, J. H. (2001). Asset Pricing, Princeton University Press. 
[13] Christie, A. A. (1982). The Stochastic Behavior of Common Stock Variances: Value, Leverage and Interest Rate Effects, Journal of Financial Economics, 10, 407-432.

[14] Daniel, K., Hirshleifer, D. and Subrahmanyam, A. (1998). Investor Psychology and Security Market Under- and Overreactions, Journal of Finance, $53,1839-1885$

[15] Decamps, J. P. and Lazrak A. (2000). A Martingale Characterization of Equilibrium Asset Price Processes, Economic Theory 15 (1), 207-213.

[16] Elerian, O., Chib, S. and Shephard, N. (2001). Likelihood Inference for Discretely Observed Nonlinear Diffusions, Econometrica, 69, 959-993.

[17] Franke, G. (1984). Conditions for Myopic Valuation and Serial Independence of the Market Excess Return in Discrete Time Models, Journal of Finance, 39, 425-442.

[18] Franke, G., Stapleton, R. C. and Subrahmanyam, M. G. (1999). When are Options Overpriced? The Black-Scholes Model and Alternative Characterisations of the Pricing Kernel, European Finance Review, 3, 79-102.

[19] Gourieroux, C. and Jasiak, J.(2001). Financial Econometrics: Problems, Models, and Methods, Princeton, University Press.

[20] He, H. and Leland, H. (1993). On Equilibrium Asset Price Processes, Review of Financial Studies, 6, 593-617.

[21] Hentschel, L. (1995). All in the Family: Nesting Symmetric and Asymmetric GARCH Models, Journal of Financial Economics, 39, 71-104.

[22] Jackwerth, J. C. (2000). Recovering Risk Aversion from Option Prices and Realized Returns, Review of Financial Studies, 13, 433-451.

[23] Johnson, T. C. (2002). Rational Momentum Effects, Journal of Finance, $57,585-608$.

[24] Lüders, E. (2002). Asset Prices and Alternative Characterizations of the Pricing Kernel, ZEW Discussion Paper No. 02-10, Centre for European Economic Research, Mannheim. 
[25] Lüders, E. and Peisl, B. (2001). How Do Investors' Expectations Drive Asset Prices? Financial Review, 36, 75-98.

[26] Mayhew, S. and Stivers, C. (2001). Stock Return Dynamics, Implied Volatility, and the Asymmetric Volatility Phenomenon, working paper, University of Georgia.

[27] Stapleton, R. C. and Subrahmanyam, M. G. (1990). Risk Aversion and the Intertemporal Behavior of Asset Prices, Review of Financial Studies, $3,677-693$.

[28] Tauchen, G. E., Zhang, H. and Liu, M. (1996). Volume, Volatility and Leverage: A Dynamic Analysis. Journal of Econometrics, 74, 177-208. 